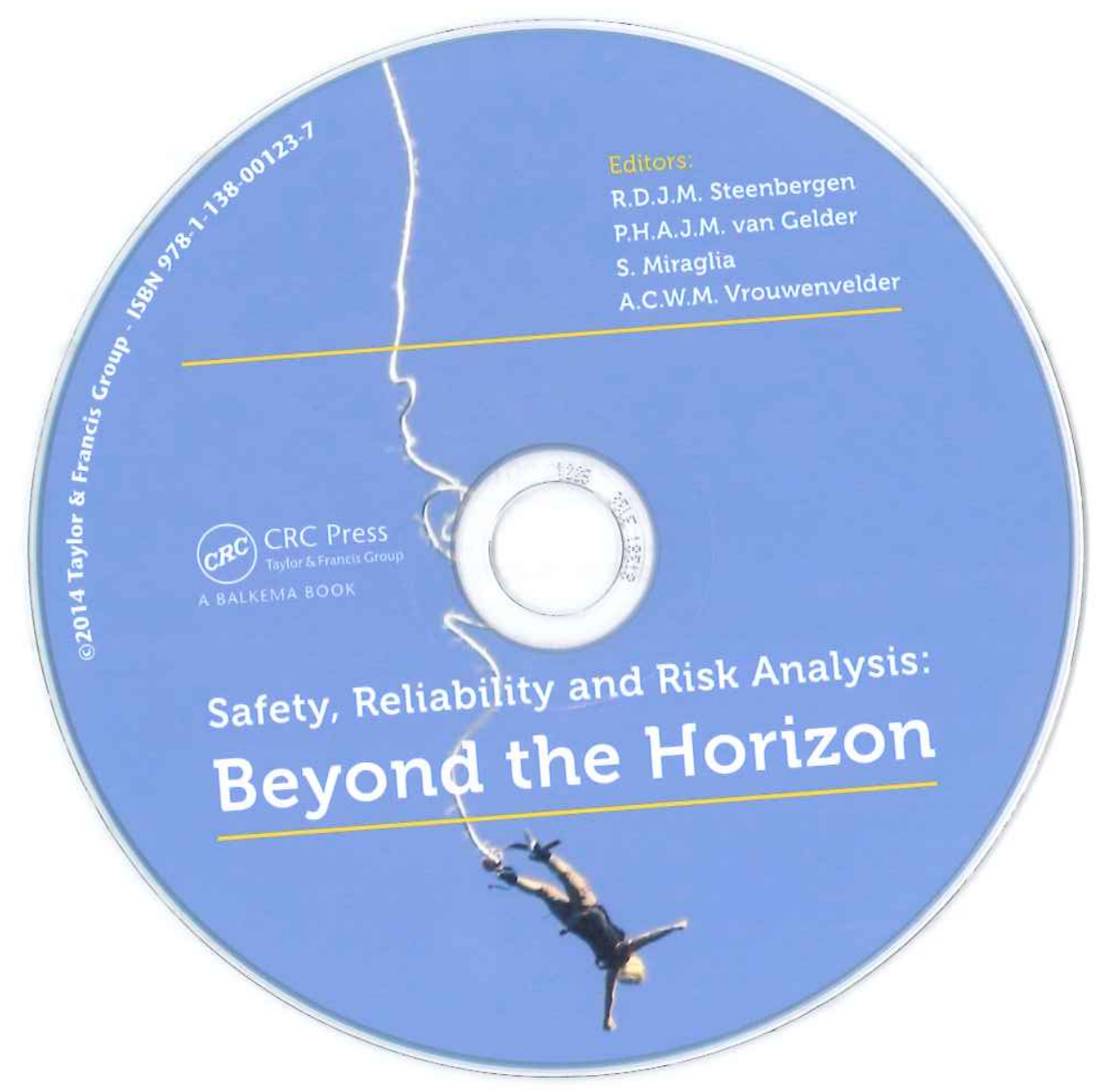




\title{
The influence of shift work in the use of hearing protection devices
}

\author{
S. Costa \& P.M. Arezes \\ Human Engineering Group, Centre for Industrial and Technology Management, \\ School of Engineering, University of Minho, Guimarães, Portugal
}

\begin{abstract}
The use of Hearing Protection Devices (HPDs) is still, to this day, the preferred measure by the companies' decision-makers to prevent workers' hearing loss. Nevertheless, it is well known that the HPDs' potential for effective protection is not fully achieved unless these devices are worn during the total amount of time they are needed, in other words, whenever workers are exposed to high levels of noise. Thus, it is of great importance to assess the HPDs' use, or non-use, by workers. Many factors have been pointed out as being responsible for the non-use of the HPDs by workers, including: lack of motivation, poor training and discomfort. The observation of workers in their real-work context has given rise to the speculation that the use of the devices depends also on the work paradigm. This paper aims at clarifying the relationship between the work done in shifts and the use of HPDs.
\end{abstract}

\section{INTRODUCTION}

The motivation for this work arose from the estimates presented by NIOSH (National Institute for Occupational Safety and Health), which indicate twenty-two million workers worldwide perform their professional activity in potentially damaging noise environments, per year. The most direct consequence of exposure to occupational noise is Noise-Induced Hearing Loss (NIHL) and, given the high prevalence of NIHL in workers of trades that present hazardous levels of noise, occupational hygiene does not appear to be effective in its purpose (NIOSH, 2011).

In terms of NIHL prevention, the use of Hearing Protection Devices (HPDs) is still, to this day, the preferred measure by the companies' decision-makers to prevent workers' hearing loss (Malchaire \& Piette 1997, Riko \& Alberti 1983, Seixas et al. 2010).

Considering the aforementioned, it seems reasonable to conclude that HPDs are not effectively protecting workers from hearing disorders. In fact, it is well known that the HPDs' potential for effective protection is not fully achieved unless these devices are worn during the total amount of time they are needed, in other words, whenever workers are exposed to high levels of noise (Brady, 1999).

Thus, it is of great importance to assess the HPDs' periods of use, or non-use, by workers.

Withal, occupational exposure to noise has also been identified as a stressor, influencing physiological processes, associated with hypertension and other illnesses (Lusk 2002, Stansfeld, 2003). These disorders are known as non-auditory effects, which are divided into two categories-physiological effects and performance effects, and include contraction of the muscles in the presence of loud noise, changes in the respiratory rhythm, in the heart beat pattern and in the diameter of the blood vessels, especially in the skin, annoyance, stress, and impaired verbal communication (Van Dijk 1986, CCOSH 2007).

Furthermore, noisy industries appear to register higher absenteeism rates. However, it is yet to be known if the latter is a consequence of psychological aversion to noise or if it results from physiological consequences of noise stress (CCOSH 2007). The relevance of the issue of the use of HPDs is further aggravated by the role of the devices in the prevention of, such as NIHL, these non-auditory disorders, rendering more positive health outcomes and constituting an important predictor of the decrease of blood pressure (Lusk 2002).

For a better sense of the importance of this issue, it should be noted that NIHL is a permanent, chronic irreversible illness, from which workers can be spared from by implementation of proper hearing conservation programs, which can include the use of HPDs - the most used resource in the prevention of NIHL (Alberti 1992, Edelson et al. 2009, Goelzer et al. 2001, Lusk 2002, Rabinowitz, 2000, Valoski 1997).

Apparently simple to solve, this problem is perpetuated in the occupational settings, due to the low rates of use of HPDs (McCullagh 2002).

The search for an explanation of this phenomenon has motivated several researches, providing a bulk of information regarding the predictors of the use of HPDs. 
Predictors are a variety of agents that exist in the occupational context and arise as factors that may be the cause of a demeanor (Arezes \& Miguel 2012, Edelson et al. 2009, Griffin et al. 2009, Kushnir et al. 2006, Lusk et al. 2004, Sbihi et al. 2010), that originate an insight (Alayrac et al. 2010), or that have influence on exposure (Abel et al. 1985, Burstyn et al. 2000, Cavallari et al. 2012).

The predictors of the use of HPDs have been identified and include: individual risk perception, subjective opinion on the company's safety climate, individual judgment on comfort, noise annoyance, education, age, trade group, seniority, perceived barriers to the use of HPDs and self-efficacy in their use, acknowledged value of the use of HPDs and deriving benefits, gender, ethnicity, dysfunctional thinking patterns and self-perceived susceptibility to hearing loss (Costa \& Arezes, 2013).

The observation of workers in their real-work context has given rise to the speculation that the use of the devices depends also on the work paradigm, besides the many factors that have been pointed out as being responsible for the use/nonuse of HPDs by workers.

Understanding the influence of the work paradigm in the use of HPDs, having been proved the hypothesis that the time percentages of use of HPDs are significantly lower for workers who perform their professional activity overnight than for workers who perform their professional activity during the day, will provide further information to assist decision-making in strategies to increase HPDs' use and decrease the risk of NIHL.

The Council Directive (EC) 2003/88/EC of 4 November 2003 concerning certain aspects of the organisation of working time defines "shift work" as a work arrangement system in shifts, by which the same work stations are sequentially occupied by different workers following a given continuous or discontinuous pattern, for example, a rotating pattern, causing workers to labour in staggered times for a certain period of time (EC 2003).

Hence, this paper aims at clarifying the relationship between the relative time of use of HPDs by shift workers and by regular workers. In addition, this study proposes to compare the percentage of time of use of HPDs by workers who labour during the night shift and the percentage of time of use of HPDs by workers who labour during the day shift.

Ultimately, the expectation is to be able to establish shift work as a predictor of the use of HPDs.

\section{MATERIALS AND METHOD}

Data regarding the use of hearing protection were collected in four companies' plants in northern
Portugal: three textile companies and a cartonnage company.

All workers, to whom the use HPDs was, at least, recommended, were invited to participate in a survey about health and hearing protection, which included a self-report item of the use of HPDs. In this item, workers were asked to indicate, in a visual analog scale, the percentage of time they actually used HPDs, in relation to the time they were supposed to wear it, per day. Suggestion to assess whether shift work affected the use of HPDs was provided by supervisors who, through observation of workers, hypothesised that the percentage of time that shift workers wore the HPDs was different from the percentage of time that regular workers wore the HPDs.

Self-report was resorted to because of its feasibility as a behaviour indicator of the use of HPDs, proved by the convenience, inexpensiveness and high validity of its use (Lusk et al. 1995).

Code numbers were used to maintain anonymity.

Of the total number of subjects of interest, 281 workers delivered their questionnaires. However, only 228 of those questionnaires have proved viable for the first part of the study (questionnaires in which workers simultaneously responded to the working shift and the self-report use of HPDs and included shift workers), and less (138 questionnaires) were proved viable for the second part of this study (questionnaires in which workers simultaneously responded to the working shift and the self-report use of HPDs, excluding the shift workers).

\section{RESULTS}

Tests were performed to check the assumption of normality of the data, which showed that the data did not follow a normal distribution. Further processing of the data was performed accordingly.

Hence, because there is only one outcome variable (percentage of time using the HPDs), which is continuous, and one predictor categorical variable with two categories, where different participants were used in each categories, Mann-Whitney tests were used to compare the results and conclude about the significance of such results.

\subsection{Part I-Shift work influence}

Demographic data in percentages, as well as mean scores by the kind of working time organisation for age and the dependent variable are displayed in Table 1.

Sample A1 consists of regular time workers $(\mathrm{n}=138)$. Ages range from 20 to 62 years old with a mean of 43.70 years old $(\mathrm{M}=43.70, \mathrm{SD}=11.23)$. Most are male $(91.3 \%)$ and $92.8 \%$ work in the 
Table 1. Demographics and percentages, including Mean Percentage of the Use of HPDs, for shift workers and for regular time workers $(n=228)$.

\begin{tabular}{|c|c|c|c|c|}
\hline \multirow{2}{*}{$\begin{array}{l}\text { Work } \\
\text { organisation }\end{array}$} & \multicolumn{2}{|c|}{ Regular $(\mathrm{n}=138)$} & \multicolumn{2}{|c|}{ Shift $(n=90)$} \\
\hline & Mean & $\mathrm{SD}$ & Mean & $\mathrm{SD}$ \\
\hline Age & 43.70 & 11.23 & 34.47 & 10.87 \\
\hline \multirow[t]{2}{*}{$\%$ use of HPDs } & 59.36 & 34.60 & 81.14 & 21.75 \\
\hline & $\%$ & $\% \mathrm{MV}^{*}$ & $\%$ & $\% \mathrm{MV}^{*}$ \\
\hline \multicolumn{5}{|l|}{ Gender } \\
\hline Male & 91.3 & 0.0 & 88.9 & 0.0 \\
\hline \multicolumn{5}{|l|}{ Education } \\
\hline$\geq 9$ th grade & 30.4 & 8.0 & 84.3 & 4.4 \\
\hline \multicolumn{5}{|l|}{ Trade } \\
\hline Textile & 92.8 & 0.0 & 95.6 & 0.0 \\
\hline
\end{tabular}

$\% \mathrm{MV}^{*}$ Percentage of missing values.

textile industry. $30.4 \%$ have an education at the ninth grade level or higher, of which only $30.92 \%$ have completed high school education, including one university graduate worker $(8 \%$ did not respond to this item). Over half of the respondents $(51.4 \%)$, self-reported their percentage of time using HPDs to be lower than $70 \%$.

Sample A2 consists of shift workers $(\mathrm{n}=90)$. Ages range from 20 to 60 years old with a mean of 34.47 years old $(\mathrm{M}=34.47, \mathrm{SD}=10.87)$. Most workers are male $(88.9 \%)$ and $95.6 \%$ work in the textile industry. $84.3 \%$ have an education at the ninth grade level or higher, of which $51.36 \%$ have completed high school education $(4.4 \%$ did not respond to this item). Almost half of the respondents (46.7\%) self-reported their percentage of time using HPDs to be equal or higher than $90 \%$. From Table 1, it can be observed that shift workers reported a higher use of HPDs $(\mathrm{M}=81.14, \mathrm{SD}=21.75)$ than workers from regular working time $(\mathrm{M}=59.36$, $\mathrm{SD}=34.60$ ). Nevertheless it is important to verify if these obtained difference is statistically significant. In fact, the Mann-Whitney test performed shows that this difference is indeed significant, i.e., the percentage of time of use of HPDs by shift workers $(\mathrm{Mdn}=89.00)$ is significantly different from workers who labour during regular time $(\mathrm{Mdn}=69.00)$, $U=4131.50, z=-4.28, p<0.01, r=-0.28, N=228$.

\subsection{Part II-Influence of the working period of the day}

Demographic data in percentages, as well as mean scores by working period for age and the dependent variable are displayed in Table 2.

Sample B1 consists of workers who labour during daytime $(n=100)$. Ages range from 20 to 62 years
Table 2. Demographics and percentages, including Mean Percentage of the Use of HPDs, for workers who labour during the day and for workers who labour overnight $(\mathrm{n}=228)$.

\begin{tabular}{|c|c|c|c|c|}
\hline \multirow[b]{2}{*}{ Working period } & \multicolumn{2}{|c|}{ Day $(n=100)$} & \multicolumn{2}{|c|}{ Night $(\mathrm{n}=38)$} \\
\hline & Mean & $\mathrm{SD}$ & Mean & SD \\
\hline Age & 42.53 & 11.72 & 46.79 & 9.28 \\
\hline \multirow[t]{2}{*}{$\%$ use of HPDs } & 63.26 & 33.77 & 49.11 & 35.09 \\
\hline & $\%$ & $\% \mathrm{MV}^{*}$ & $\%$ & $\% \mathrm{MV}^{*}$ \\
\hline \multicolumn{5}{|l|}{ Gender } \\
\hline Male & 88.0 & 0.0 & 100.0 & 0.0 \\
\hline \multicolumn{5}{|l|}{ Education } \\
\hline$\geq 9$ th grade & 35.0 & 8.0 & 18.5 & 7.9 \\
\hline \multicolumn{5}{|l|}{ Trade } \\
\hline Textile & 91.0 & 0.0 & 97.4 & 0.0 \\
\hline
\end{tabular}

$\% \mathrm{MV}^{*}$ Percentage of missing values.

old with a mean of 42.53 years old $(\mathrm{M}=42.53$, $\mathrm{SD}=11.72)$. Most are male $(88.0 \%)$ and $91.0 \%$ work in the textile industry. $35.0 \%$ have an education at the ninth grade level or higher, of which only $31.43 \%$ have completed high school education, including one university graduate worker $(8 \%$ did not respond to this item). Half of the respondents $(50.0 \%)$ self-reported their percentage of time using HPDs to be lower than $80 \%$.

Sample B2 consists of workers who labour overnight $(\mathrm{n}=38)$. Ages range from 21 to 59 years old with a mean of 46.79 years old $(\mathrm{M}=46.79$, $\mathrm{SD}=9.28)$. All workers are male $(100.0 \%)$ and $97.4 \%$ work in the textile industry. $18.5 \%$ have an education at the ninth grade level or higher, of which only $28.65 \%$ have completed high school education ( $7.9 \%$ did not respond to this item). Over $60 \%$ of the respondents $(60.5 \%)$ self-reported their percentage of time using HPDs to be lower than $60 \%$. Table 2 , shows that workers who labour during daytime reported a higher use of HPDs $(\mathrm{M}=63.26$, $\mathrm{SD}=33.77)$ than workers from regular working time $(\mathrm{M}=49.11, \mathrm{SD}=35.09)$. Nevertheless it is important to verify if these obtained difference is statistically significant. In fact, the Mann-Whitney test performed shows that this difference is indeed significant, i.e., the percentage of time of use of HPDs by workers who labour during daytime $(\mathrm{Mdn}=78.00)$ is significantly different from workers who labour overnight $(\mathrm{Mdn}=50.00), U=1441.50$, $z=-2.19, p<0.05, r=-0.19, N=138$.

\section{DISCUSSION}

The objective of this research was to clarify the relationship between the working time organisation 
and the use of HPDs, as well as to compare the use of HPDs between workers who labour overnight and workers who labour during daytime. These data would ultimately allow for the establishment of whether the shift work was or was not a predictor of the use of HPDs.

Results show that there is a significant difference between the relative time of use of HPDs between both groups that constitute the two types of working time organisation. In fact, shift workers' percentage time of use of HPDs are higher than regular time workers'. Moreover, the organisation of the working time has a small to medium effect over the use of HPDs.

Also, it can be observed that workers who perform their professional activity overnight use significantly less their HPDs, relatively to workers who perform their professional activity during the day. This means that the shift work has an influence in the use of HPDs, even though it represents a small effect.

However, this is not a surprising result, and can be explained by the fact that night shifts are more "relaxed", i.e., less likely to be subject to audits, and less supervised. Moreover, a part of this result may be explained by the fact that men exclusively form sample B2, and gender is a known predictor of the use of HPDs.

On the other hand, as stated before, there are many predictors to the use of HPDs, so it seems reasonable that some of them will have less impact in the use of HPDs' behaviour than others, and the work shift is one of the predictors with less effect.

\section{CONCLUSIONS}

Taking into account the results obtained with respect to what has been proposed to assess with this work, it is concluded that the work shift influences the use of HPDs and that shift workers' relative use of HPDs is significantly higher than workers in regular working time organisations.

In addition, it is concluded that, overnight, the percentage of time of use of HPDs is significantly smaller than the percentage of time of use of HPDs during the daytime.

These results support the hypothesis posed by supervisors that, in fact, the work paradigm affects the use of HPDs.

Also, it is concluded that the work shift is a factor that explains the use of HPDs', thus posing as a predictor of such behaviour.

\section{FUTURE WORK}

To better fundament this study, a strategy of observation through sampling of the workers is being developed, that scans all work paradigms (shifts).

\section{ACKNOWLEDGEMENTS}

The author S. Costa expresses her acknowledgments for the support given by the Portuguese Foundation for Science and Technology (FCT) through the $\mathrm{PhD}$ grant SFRH/BD/69161/2010.

\section{REFERENCES}

Abel, S.M., Kunov, H., Pichora-Fuller, M.K. \& Alberti, P.W. 1985. Signal detection in industrial noise: Effects of noise exposure history, hearing loss, and the use of ear protection. Scandinavian Audiology 14(3): 161-173.

Alayrac, M., Marquis-Favre, C., Viollon, S., Morel, J. \& Le Nost, G. 2010. Annoyance from industrial noise: indicators for a wide variety of industrial sources. The Journal of the Acoustical Society of America 128: 1128.

Alberti, P.W. 1992. Noise induced hearing loss. British Medical Journal 304(6826): 522.

Arezes, P.M. \& Miguel, A.S. 2012. Assessing the use of hearing protection in industrial settings: A comparison between methods. International Journal of Industrial Ergonomics. Retrieved August 15, 2012, from http://www.sciencedirect.com/science/article/pii/ S0169814112000637.

Brady, J. 1999. Training to promote worker's use of hearing protection: The influence of work climate factors on training effectiveness. PhD Thesis, Michigan State University: Michigan, 274 pgs.

Burstyn, I., Kromhout, H. \& Boffetta, P. 2000. Literature review of levels and determinants of exposure to potential carcinogens and other agents in the road construction industry. American Industrial Hygiene Association Journal 61(5): 715-726.

Cavallari, J.M., Osborn, L.V., Snawder, J.E., Kriech, A.J., Olsen, L.D., Herrick, R.F. \& Mcclean, M.D. 2012. Predictors of airborne exposures to polycyclic aromatic compounds and total organic matter among hot-mix asphalt paving workers and influence of work conditions and practices. Annals of Occupational Hygiene 56(2): 138-147.

CCOSH. 2007. CCOSH-Noise-Non-Auditory Effects Topic. In Canadian Centre for Occupational Health and Safety. Retrieved April 4, 2013 from: http://www. ccohs.ca/oshanswers/phys_agents/non_auditory.html.

Costa, S., \& Arezes, P. 2013. On the nature of hearing protection devices usage prediction. Proceedings from SHO 2013: The International Symposium on Occupational Health and Hygiene, Guimarães, Portugal.

Council Directive (EC) 2003/88/EC of 4 November 2003 concerning certain aspects of the organisation of working time.

Edelson, J., Neitzel, R., Meischke, H., Daniell, W., Sheppard, L., Stover, B. \& Seixas, N. 2009. Predictors of hearing protection use in construction workers. Annals of Occupational Hygiene 53(6): 605.

Goelzer, B., Hansen, C.H. \& Sehrndt, G.A. 2001. Occupational exposure to noise: evaluation, prevention and control. Federal Institute for Occupational Safety and Health. 
Griffin, S.C., Neitzel, R., Daniell, W.E. \& Seixas, N.S. 2009. Indicators of hearing protection use: self-report and researcher observation. Journal of Occupational and Environmental Hygiene 6(10): 639-647.

Kushnir, T., Avin, L., Neck, A., Sviatochevski, A., Polak, S. \& Peretz, C. 2006. Dysfunctional thinking patterns and immigration status as predictors of hearing protection device usage. Annals of Behavioral Medicine 32(2): 162-167.

Lusk, S.L., Eakin, B.L., Kazanis, A.S., \& McCullagh, M.C. 2004. Effects of booster interventions on factory workers' use of hearing protection. Nursing Research 53(1): 53-58.

Lusk, S.L., Hagerty, B.M., Gillespie, B., Caruso, C. 2002. Chronic Effects of Workplace Noise on Blood Pressure and Heart Rate. Archives of Environmental Health: An International Journal 57(4): 273-281.

Lusk, S.L., Ronis, D.L., \& Baer, L.M. 1997. Gender differences in blue collar workers' use of hearing protection. Women \& Health 25(4): 69-89.

Malchaire, J. \& Piette, A. 1997. A comprehensive strategy for the assessment of noise exposure and risk of hearing impairment. Annals of Occupational Hygiene 41(4): 467.

McCullagh, M., Lusk, S.L. \& Ronis, D.L. 2002. Factors Influencing Use of Hearing Protection Among Farmers: A Test of the Pender Health Promotion Model. Nursing Research 51(1): 33-39.
NIOSH. 2011. CDC-Noise and Hearing Loss Prevention-Facts and Statistics-NIOSH Workplace Safety and Health Topic. In Centers for Disease Control and Prevention. Retrieved February 25, 2013 from: http:// www.cdc.gov/niosh/topics/noise/.

Rabinowitz, P.M. 2000. Noise-induced hearing loss. American Family Physician 61(9): 2759-2760.

Riko, K. \& Alberti, P. 1983. Hearing protectors: a review of recent observations. Journal of Occupational and Environmental Medicine 25(7): 523.

Sbihi, H., Teschke, K., Macnab, Y.C. \& Davies, H.W 2010. Determinants of use of hearing protection devices in Canadian lumber mill workers. Annals of Occupational Hygiene 54(3): 319-328.

Seixas, N.S., Neitzel, R., Stover, B., Sheppard, L., Daniell, B., Edelson, J., \& Meischke, H. 2010. A multicomponent intervention to promote hearing protector use among construction workers. International Journal of Audiology, 50 Suppl 1:S46-56.

Stansfeld, S.A. \& Matheson, M.P. 2003. Noise pollution: non-auditory effects on health. British Medical Bulletin 68(1): 243-257.

Valoski, M.P. 1997. Reported noise-induced hearing loss among miners 12(12): 1055-1058.

Van Dijk, F.J.H. 1986. Non-auditory effects of noise in industry-II A review of the literature. International Archives of Occupational and Environmental Health 58(4): 325-332. 